\title{
Fault Detection of Power Electronic Circuit using Wavelet Analysis in Modelica
}

\author{
Jianbo Gao*, Yang Ji**, Johann Bals**, Ralph Kennel* \\ *Technische Universität München \\ Arcisstr. 21, 80333 Munich, Germany \\ michael.gao@tum.de,ralph.kennel@tum.de \\ ** German Aerospace Center \\ Muenchner Str. 20, 82234 Wessling, Germany \\ yang.ji@dlr.de,johann.bals@dlr.de
}

\begin{abstract}
In more electric aircrafts (MEA) the electric power network is important for the reliability. To prevent severe faults it is the key issue to identify the faults in the early stage before a complete failure happens. In this paper an early stage fault detection method using wavelet multi-resolution analysis (MRA) for a regulated buck DC-DC converter is studied. Specifically, the electrolyte input capacitor is diagnosed. The study was carried out using simulation with Modelica / Dymola. The fault features that were extracted from different levels of wavelet decomposition provided clear information for both fast and slow occurring faults. This method showed significant advantages compared with filter techniques. It is concluded that wavelet transform is a suitable tool for early stage fault detection of the power electronics in MEA. In addition, the simulation language Modelica provides a convenient possibility for the quick design of fault detection strategy.
\end{abstract}

Keywords: power electronics; $D C$-DC converter; fault detection; wavelet; Modelica; Dymola

\section{Introduction}

\subsection{Motivation}

The concept of More Electric Aircraft (MEA) is attracting increasing interest in the aircraft industry not only because of its potential in energy optimization, but also due to its significant advantages concerning weight, maintenance requirements, liability and passenger comfort [1]. For this, the electrical power distribution network is playing a more important role and facing increasing challenges in the prognosis and accurate localization of faulty units in an even more complex power network. In order to obtain maximum flight reliability and minimum maintenance efforts, advanced failure analysis technologies shall be applied to ensure correct and quick fault detection and isolation. It is well known that an output voltage regulated DC/DC power converter supplying constant power loads could de-stabilize the network stability due to the degraded performance of its input filter. The sensitivity study of input filter parameters concerning the network stability addressed in [2] reveals that the observation of degraded degree of the capacitor in the input filter can significantly increase the network reliability.

\subsection{State of the art}

Reviewing the considerable development of fault diagnosis techniques and many successful applications attached to them in the last time [3] [4] [5], power systems keep a challenge for fault detection. For overcoming this challenge intelligent methods like artificial neural networks have shown their possibilities in this field [6]. Besides, the analytical model based technology is also obtaining more attention [7] [8] [9].

Signal-based methods, e.g. Fourier transform and wavelet transform, also provide other possibilities to perform the fault detection and isolation. With the rash development of the new mathematical tool, wavelet transform [10], a great amount of studies have been done in different fields for fault detection. Some attempts have also been made in power electronics for fault detection [11] [12]. The implementation of wavelet transform for the post processing of Modelica simulation data has been seen, for example, in a study of vehicle steering, where wavelet transform was carried out in the software Matlab for calculating power spectra [13]. 


\subsection{Main contributions}

Modelica was developed as a free, object-oriented and equation-based modeling language. It has significant benefits such as easy reusability of models and multi-domain modeling capability. In combination with the simulation environment Dymola, a convenient platform is provided to the complete modelbased design and the integration of MEA systems [14]. In contrast to the excellent performance in modeling and simulation, Modelica only supports limited signal analysis features [15], which are actually crucial for the fault analysis and virtual testing activities in the verification and validation phase of the system development.

This work focuses on the fast design of a fault detection strategy of on board power supply units in MEA with wavelet transform using Modelica simulation. To realize this, a wavelet library for Modelica is being developed. Multi-Resolution Analysis (MRA) of wavelet technology is applied to detect the failure of electrolyte capacitors in a very early stage.

In addition, the design process using Modelica simulation shows high flexibility and efficiency. It is possible to identify the most important failure features and helps to design a effective fault detection strategy within only a short time.

\section{Wavelet transform}

\subsection{Definition}

Wavelet transform could be considered as a further development of Fourier transform, or more precisely, of short time Fourier transform (STFT) [16]. Using STFT, people try to localize the signal changing with time by selecting suitable time window. This transformation, however, is limited in timefrequency resolution capability due to the uncertainty principle. Wavelet transform overcomes this problem. This transform is defined as [10]:

$\left[W_{\psi} f\right](a, b)=\frac{1}{\sqrt{|a|}} \int_{-\infty}^{\infty} \overline{\psi\left(\frac{t-b}{a}\right)} f(t) \mathrm{d} t$.

It is described as the wavelet transform of the square-integrable function, $f$, using wavelet function, $\psi$, at dilation (or scale), $a$, and position (or translation), $b$. The bar above function, $\psi$, stands for conjugation. For the given $a$ and $b$, the transform result is a single real number, a wavelet coefficient.

Obviously wavelet transform is the integral of the multiplication of the signal, $f$, with a wavelet function, $\psi$. It has the same form as the STFT. However, not like STFT, where only sine and cosine functions are used for the transformation, wavelet transform uses different wavelet functions, which can be selected according to the specific problems from a principally unlimited set. Nevertheless, the wavelet function must fulfill some conditions; namely, it must be an orthonormal function. The precise mathematical description of orthonormality is easily found in almost every book about wavelet transform, e.g. [10], and is not repeated here.

Parameter, $a$, defines the width and height of the wavelet function, $\psi$. A smaller scale, $a$, makes $\psi$ narrower; thus the wavelet represents fast changes and the transform focuses on the high frequency components of the signal. Parameter, $b$, shifts the wavelet function along the time axis, so that the transform represents different locations of the signal. Using different values of scale, $a$, and position, $b$, it is able to observe the signal at different position and in different frequency range with only one transformation. Thanks to these special properties, wavelet transform is especially suitable for analyzing changing processes.

Two forms of wavelet transform are available. They are continuous wavelet transform (CWT) and discrete wavelet transform (DWT). In CWT both scale and position parameters are continuous real values. CWT expresses the signal changes in continuous details. It is more suitable for visual examination. In this work only DWT is used, which will be described in more detail in the next section.

\subsection{Discrete wavelet transform}

In DWT only discrete values of the scale and location parameters are used. The values are selected in a discrete form, namely

$\psi_{j, k}=2^{-\frac{j}{2}} \psi\left(a^{-j} x-k b\right)$,

where $a>1, b>0$ and $j, k \in \boldsymbol{Z}$. The transform results, i.e. the wavelet coefficients, are therefore also discrete.

In the numeric calculation of DWT, an extra scaling function, in addition to the wavelet function, is used to carry out a complete and efficient DWT. The scaling function represents the low frequency components of the signal. It is orthogonal to its own discrete translations and to all wavelet functions. The wavelet and the scaling functions with the discrete scaling and translation parameters build a complete orthogonal basis of the Hilbert space. The DWT is thus another representation of the time signal.

As an example, Figure 1 shows the form of the third order Daubechies scaling and wavelet functions and their Fourier transforms [17]. 


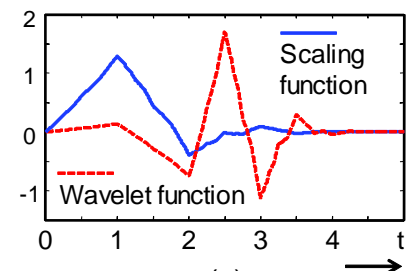

(a)

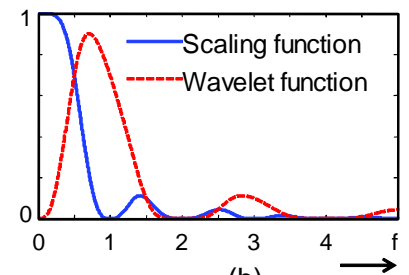

(b)
Figure 1: The third order Daubechies scaling and wavelet functions (a) and their Fourier transforms (b)

From the Fourier transforms it can be seen that the scaling function covers lower frequency range while the wavelet function stretches in a higher frequency range. From this point of view, DWT is actually the division of the time signal into different frequency bands. Thus, it is straightforward to understand that the calculation of DWT is realized using filter banks. In inverse DWT the calculation is similar. This process is illustrated in Figure 2.

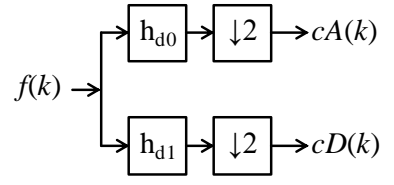

DWT

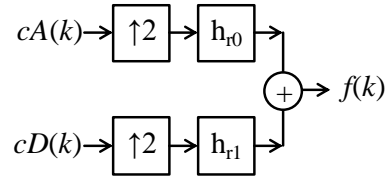

Inverse DWT
Figure 2: DWT and inverse DWT calculation using filter banks

DWT transforms the original sequence in two new series:

(1) the approximation coefficients, $c A(k)$, representing the low frequency components, obtained using the low pass filter for decomposition, $\mathrm{h}_{\mathrm{d} 0}$, and

(2) the detail coefficients, $c D(k)$, representing the high frequency components, obtained using the high pass filter for decomposition, $\mathrm{h}_{\mathrm{d} 1}$.

The symbol $\downarrow 2$ means down sampling. The operation is to delete one from every two adjacent coefficients, in order to remove the redundant information. The inverse DWT carries out the reversed operation. The operator, $\uparrow 2$, expands a coefficient series by inserting a zero between every two adjacent elements. After that the two series pass through the filter bank, and added together to get the original signal.

\subsection{Multi-resolution analysis}

Considering the DWT process shown in Figure 2, sequence, $c A(k)$, which represents the low frequency components can be further divided into a lower frequency part and a higher frequency part inside the frequency range of $c A(k)$. This process is repeated and a series of coefficient sequences representing different frequency ranges is obtained, as shown in Figure 3:

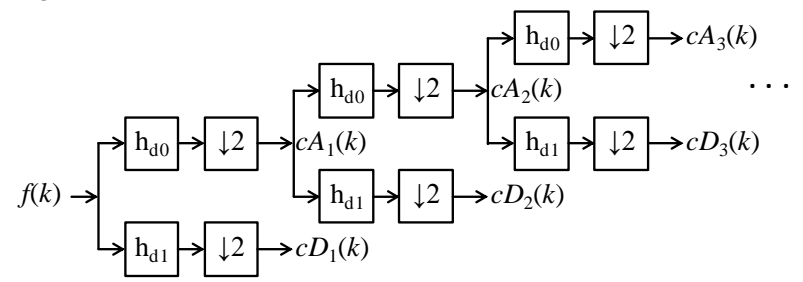

Figure 3: Multi-resolution analysis using DWT

This is the wavelet multi-resolution analysis (MRA). The output of this operation, $c D_{1}, c D_{2}, \ldots$, $c D_{\mathrm{n}}$ and $c A_{\mathrm{n}}$, are different levels of DWT coefficients, representing the signal components from higher to lower frequencies. Here the original signal is treated as the lowest level of approximation coefficients. This analysis provides a convenient tool to observe different frequency components of the signal depending on time.

\subsection{Wavelet analysis for fault detection}

Wavelet transform is a powerful tool in signal processing for the detection of changing events. This feature is suitable for fault detection since a fault in a system can be treated as a deviation compared to the normal state.

When a fault occurs, specific changes will appear in the sensor signal. Usually, it is known that the fault signal is located in a certain frequency range, but the exact frequency is often unknown or not constant. This problem can be handled with wavelet MRA. For that, the signal containing fault information is firstly decomposed in several levels. And in one or more levels, where the fault signal frequency is located, faults features will be observed.

\section{Wavelet fault detection in a MEA power network system}

Based on the properties of wavelet transform in signal processing, this new mathematic tool is used for fault detection in a MEA power network system in this study. Specifically, the MRA is used here to detect the capacitance drop of the input capacitor in a DC-DC buck converter for the early stage failure.

\subsection{The problem}

The buck converter is described with the diagram shown in Figure 4. 


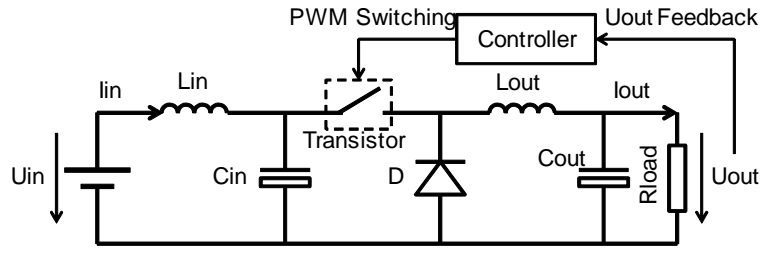

Figure 4: Diagram of the Buck converter under study

The converter operates in constant load power mode by keeping a constant output voltage, which is adjusted through the duty cycle of the pulse width modulation (PWM) switching signal, which operates with a constant frequency. Based on the converter property the system is sensible to the value changes of the components on the input side, where the input capacitor, $\mathrm{Cin}$, is especially critical because it is usually an electrolyte type, which has significantly lower feasibility and shorter lift time compared with other components. Base on this reason, the early fault detection is focused on Cin.

Four parameters of the circuit can be conveniently measured by voltage and current sensors. They are, referring to Figure 4, Uout, Iout, Iin and Uin, ordered from higher to lower ease of availability. Since the load is pure resistive constant load, the output current signal will contain the same information as the output voltage. And Uout will be used any way for the controller as feedback, the equipment of a sensor for output current is therefore not necessary, at the least for fault detection.

Since a stable output voltage is the control objective of the circuit, the influence of $\mathrm{Cin}$ would be compensated by the controller very quickly. As a consequence only very few fault information would be propagated to the output side. The fault detection using Uout is therefore not feasible.

The input voltage is not a good signal for fault detection, too, since it actually measures the input power supply voltage, which is normally a voltage source with very low impedance and thus hardly be influenced by $\mathrm{Cin}$.

The input current is the last possibility; and it is actually also a suitable signal for fault detection. The reason is, for example, if its capacitance drops, it means the energy capacity of the input circuit is reduced. In order to keep a constant energy flow to the load, which is regulated by the controller, Cin would have to be charged and discharged more deeply. This will be reflected in the input current with larger fluctuation. This estimation will be showed later in the result section.

If an electrolyte capacitor approaches it life end, its capacity would reduce slowly within a certain time. However, sharp reduction or changing of ca- pacitance might also occur. For fault detection, especially for early stage fault diagnosis, both stepping type and slow changing of capacitor fault should be considered.

\subsection{Extraction of fault information}

The first step is the extraction of the fault information from the sensor signals. Supposing the measured signal is

$f(k)=x(k)+g(k)$,

where $x(k)$ is the signal in normal operation state, and $g(k)$ the additional signal in fault condition.

Using wavelet technology, the sensor signal $f(k)$ is decomposed with MRA using wavelet function, $\psi$, to obtain wavelet coefficients in $n$ levels:

$$
\begin{aligned}
D W T_{\psi}\{f\}= & A_{n}\{f\}+\sum_{i=1}^{n} D_{i}\{f\}, \\
= & A_{n}\{x\}+A_{n}\{g\} \\
& +\sum_{i=1}^{n}\left(D_{i}\{x\}+D_{i}\{g\}\right)
\end{aligned}
$$

where $D_{i}\{$.$\} represents the detail coefficients, and$ $A_{i}\{$.$\} stands for approximation coefficients. The term$ $D_{i}\{$.$\} with smaller index, i$, represents higher frequency components, namely faster changing signals.

The signal, $x$, in the normal operation condition is composed of the average value of the battery current, which changes very slow, and the ripples caused by the PWM controlling, which have a constant frequency defined by the controller. The slower components are transformed to the approximation coefficients, $A_{n}\{x\}$; and the components with PWM frequency, which are higher frequency components, is transformed to the very low level of $D_{i}\{x\}$.

On the other hand, the fault signal, i.e. the information of the reduction of Cin, is reflected by the fluctuation of the input current. As it is known that the fluctuation frequency is actually the PWM frequency, most of the fault information is contained in the PWM components, which means the lower levels of detail coefficients, $D_{i}\{g\}$. Depending on the fault occurrence rate, complex fluctuation could take place. This information will be carried by the PWM frequency, too, but its own frequency components are visible in other levels of $D_{i}\{g\}$.

In any circumstance few information will be present in very low frequency range, i.e. in $A_{n}\{g\}$. Therefore, we can extract the fault information from the input current signal simply by isolating some levels of detail coefficients, $D_{i}\{f\}$. Of course, the PWM information will also be involved. It has to be removed before the faults can be identified. Since this frequency is known and it always has a very high value, these components can be easily suppressed with low pass filter or band stop filter. 


\subsection{Fault identification}

After all irrelevant information is excluded the final fault information is represented with a single value, changing according to the failure rate. The fault can be simply identified by comparing it with a known threshold.

\section{Design of a fault detection strategy using Modelica}

Because of the aforementioned superior properties, Modelica simulation and wavelet transform were selected for the quick design of a fault detection strategy for the power system in MEA. Since wavelet transform is not available in the standard Modelica libraries, a solution have to be found. A seemingly direct solution would be the use of a second software tool, which provides wavelet analysis capacity, such as Matlab from MathWorks. Some practical reasons were faced, however. Firstly, the use of such commercial software requires expensive licenses. Secondly, a single program both for simulation and data analysis is very desirable during the work in order to have an integrated working process and to avoid interfacing between two programs. It is therefore more favourable to have the wavelet analysis inside Modelica. In addition, this brings further advantages in that the library can be a common tool of Modelica, so that higher work efficiency will be achieved in a long term.

\subsection{Model of the power supply}

The buck converter shown in Figure 4 is realized with a Dymola model in Figure 5.

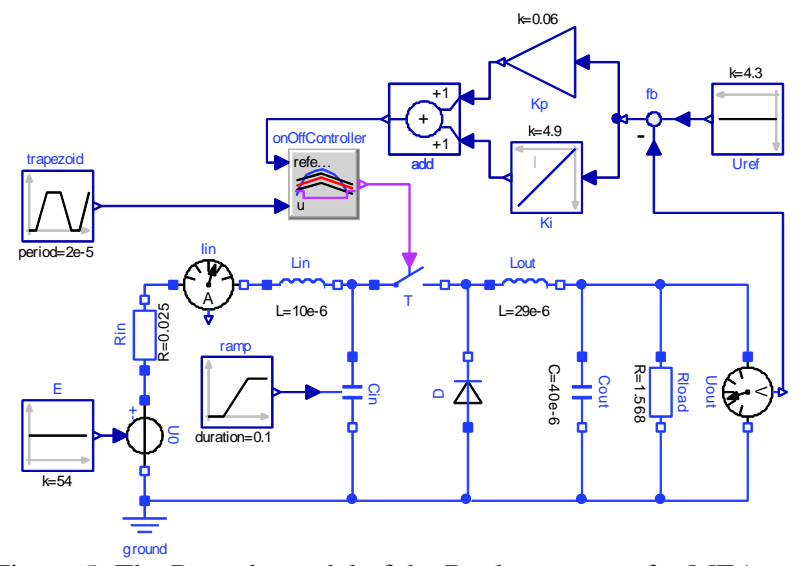

Figure 5: The Dymola model of the Buck converter for MEA

The voltage controller is a proportional-integral regulator. The output voltage is set as $4.3 \mathrm{~V}$. The PWM frequency is defined with the trapezoidal source as $50 \mathrm{kHz}$. The component fault is simulated by reducing the value of the input capacitor, $C$ in, with a ramp source. By setting the ramp, different changing rate of the component value can be simulated. The input current is measured by a current sensor, which is explicitly put in the model only for clarity, since the current values could actually be read out directly from the corresponding components, e.g. Rin or Lin. Other parameters are listed in Table 1

Table 1: Parameters of the buck converter

\begin{tabular}{|l|l|l|}
\hline Parameter & Description & Value \\
\hline$E$ & Voltage source & $54 \mathrm{~V}$ \\
\hline Uref & Reference output voltage & $4.3 \mathrm{~V}$ \\
\hline Lout & Output inductance & $29 \mu \mathrm{H}$ \\
\hline Cout & Output capacitance & $40 \mu \mathrm{F}$ \\
\hline Rload & Load resistance & $1.568 \Omega$ \\
\hline Lin & Input filter inductance & $10 \mu \mathrm{H}$ \\
\hline Cin & Input filter capacitance & $10 \mu \mathrm{F}$ \\
\hline Rin & Input resistance & $0.025 \Omega$ \\
\hline$K p$ & Propotional controller gain & 0.06 \\
\hline$K i$ & Integral controller gain & 4.9 \\
\hline
\end{tabular}

\subsection{Wavelet transform in Modelica}

The structure of the wavelet toolbox developed for Modelica is shown in Figure 6. At the moment of this report the wavelet library is under development within the frame of Clean-Sky project organized by European Union. It is expected to be a general Modelica library with wavelet transform and some related functionality for different signal processing purposes. This library is used for post processing of the simulation result data and cannot be embedded into simulation models. So far the core library functions have been realized and wavelet DWT and MRA can be implemented for the reported work.

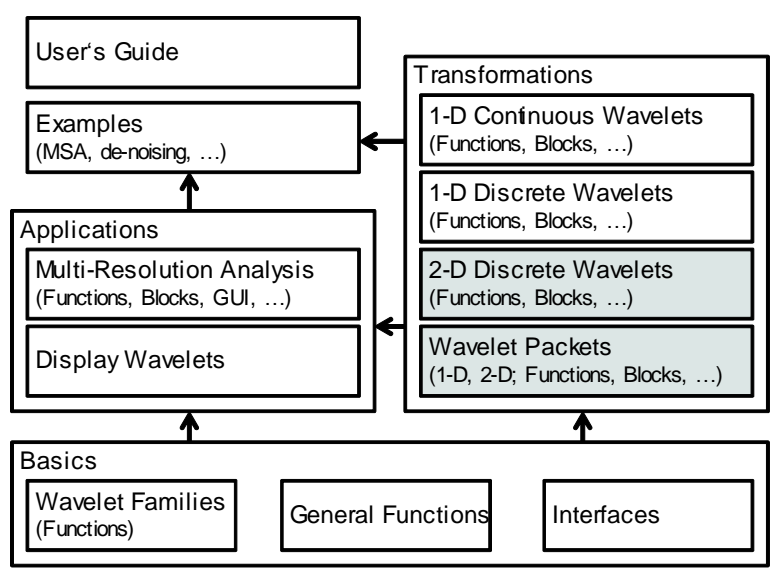

Figure 6: Structure of the intended Modelica wavelet library (the functions with dark background will be designed depending on the work process) 


\subsection{Process for simulation and fault detection}

As mentioned in the problem description (section 3.1) the input current signal is used for fault detection. At first the model will be simulated with different reduction rates of the Cin capacitance. The reduction rates were selected between 1 and $100 \mathrm{~ms}$ with a capacitance drop from 10 to $8 \mu \mathrm{F}$, corresponding to $20 \%$ capacitance loss. With this fault level the system can still operate normally. However, a $20 \%$ reduction indicates a high possibility of a complete failure of the capacitor in the near future. The reduction is applied at $0.1 \mathrm{~s}$ after the startup of the simulation so that the system can achieve a stable state before the faults could occur.

After simulation, the data segment containing the fault event will be read out from the simulation result data file. It is converted to equidistant time series with a sampling rate of $200 \mathrm{kHz}$. Equidistant sampling is the requirement of wavelet transform and most other signal processing methods. The fault detection process is illustrated in Figure 7.

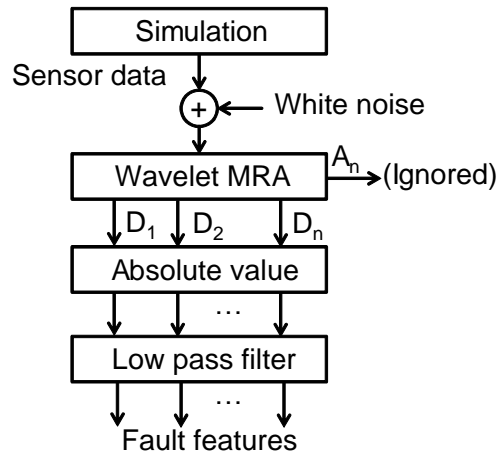

Figure 7: Process for simulation and fault detection using wavelet transform

To simulate the real world, a white noise signal with normal distribution is added on the input current signal. After that, MRA is applied on the data. The wavelet function used here was the third order Daubechies function shown in Figure 1. The detail coefficients in the DWT result are extracted and their absolute values are calculated since only the magnitude of the DWT coefficients contains fault information. To remove the high frequency PWM component, second order Butterworth low pass filter is used. The filter cut off frequency is set as $0.5 \mathrm{kHz}$, much lower than that of the PWM frequency, in order to suppress a large part of the noise signal, too. After this step, different fault features can be visually identified and suitable fault detection methods can be established.

\subsection{Results}

The tests with different parameters were carried out. Figure 8 and Figure 9 give two examples with slow and fast changing faults, respectively. Since all fault features mainly present in the first three levels of the wavelet decomposition, only these coefficients are shown in the figures.

It was noticed that the fault features differed significantly between fast and slow changing rates. For the changing rates faster than $5 \mathrm{~ms}$, a pulse feature appeared in almost every level of the wavelet detail coefficients. This is well seen in Figure 8, where $20 \%$ capacitance drop took place within $1 \mathrm{~ms}$. In the first MRA level the feature magnitude changed from 55 to $70 \mathrm{~mA}$ with the fault. In the other two higher levels the fault event was extra present with pulses. The magnitude of the pulses increased with the changing rate. The pulses appeared in other higher decomposition level, too. However, in level three it was significantly higher than those in other levels. For the fault with $1 \mathrm{~ms}$ dropping time, as shown in Figure 8 , the pulse peak reached almost $100 \mathrm{~mA}$ in level 3. The simulation showed that for the dropping time up to $5 \mathrm{~ms}$, the peak height reduced linearly to about $20 \mathrm{~mA}$.

For the changing rates slower than $5 \mathrm{~ms}$, the fault features were only significant in the lowest level of the wavelet MRA coefficients. Figure 9 shows an example, where the $20 \%$ capacitance drop took 100 ms time. Now only the first wavelet decomposition level contained a fault feature: the feature magnitude changed from about 55 to $70 \mathrm{~mA}$ linearly with the reduction of the capacitance. This was the same as the level 1 feature in fast changing faults. For even lower changing rates this linear relationship was always present. Not like in the case of fast changing fault, no significant features were observed in other decomposition levels.

Based on the simulation study, the following fault detection strategy can be defined for early stage fault detection of the input capacitor, Cin:

(1) In wavelet decomposition level one, if the feature magnitude exceeds $70 \mathrm{~mA}$, a capacitance reduction of $20 \%$ of $\mathrm{Cin}$ is detected.

(2) In wavelet decomposition level three, if the pulse value exceeds $20 \mathrm{~mA}$, the event implies a fast drop of the Cin capacitance. The pulse peak value could be used to estimate the capacitance changing rate. 

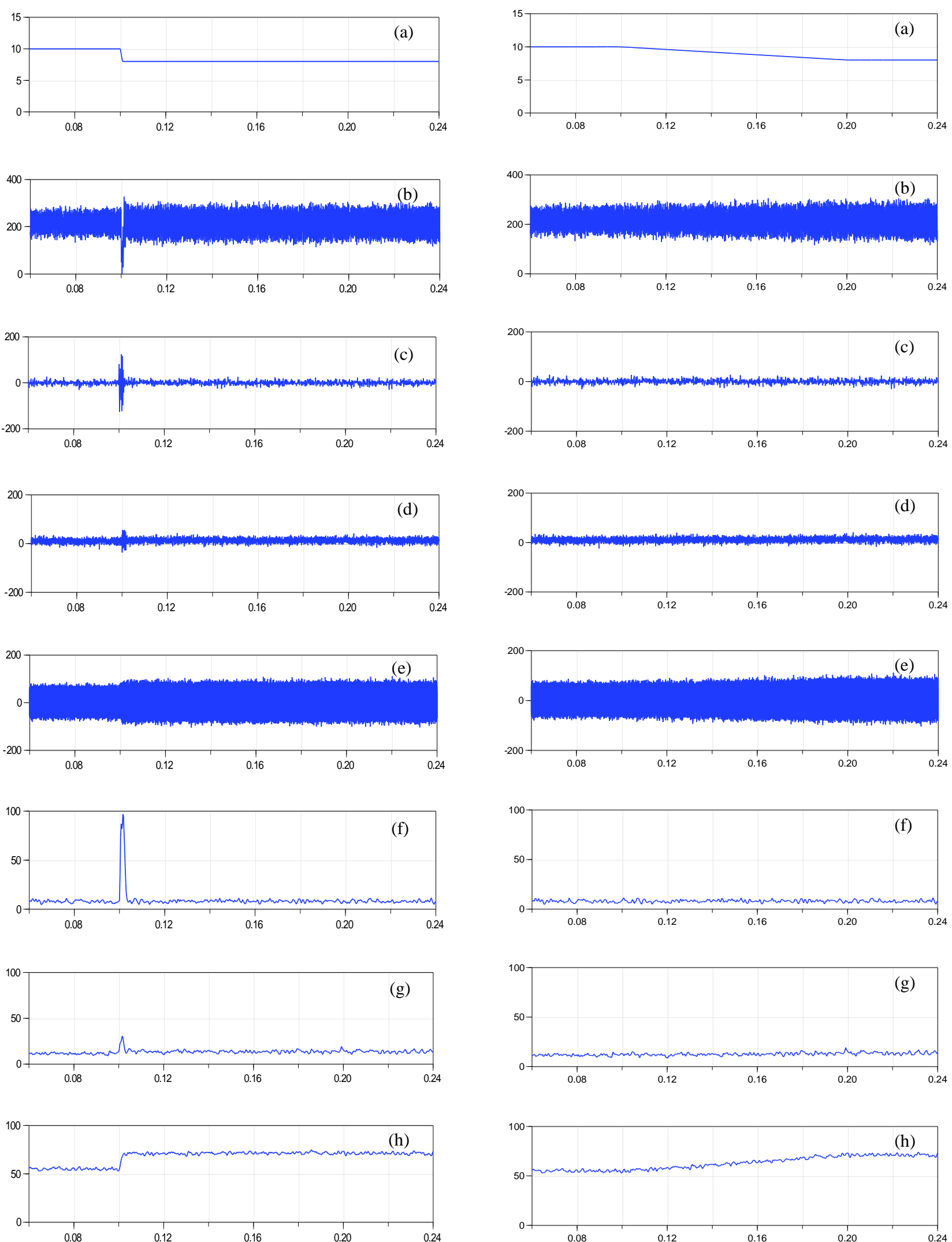

Figure 8: Signals in the detection of a capacitance drop fault within $1 \mathrm{~ms}$ using wavelet transform. (a) Cin capacitance $(\mu \mathrm{F})$; (b) input current signal, Iin (mA); (c-e) detail wavelet coefficients in MRA from Level 3 to level 1 (mA); (f-h) fault features contained in detail wavelet coefficients of level 3 to level 1 $(\mathrm{mA})$.

Figure 9: Signals in the detection of a capacitance drop fault within $100 \mathrm{~ms}$ using wavelet transform. (a) Cin capacitance $(\mu \mathrm{F})$; (b) input current signal, Iin (mA); (c-e) detail wavelet coefficients in MRA from Level 3 to level 1 (mA); (f-h) fault features contained in detail wavelet coefficients of level 3 to level 1 $(\mathrm{mA})$. 


\subsection{Comparison with filter technique}

The wavelet fault detection method was compared with the traditional filter technique, which was processed according to the procedure as shown in Figure 10.

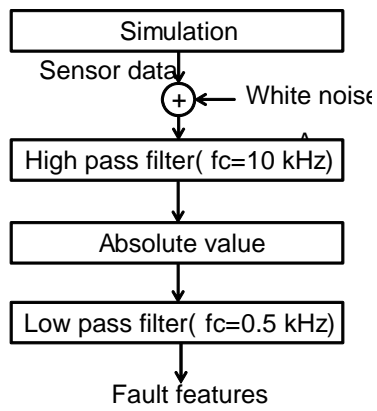

Figure 10: Process for simulation and fault detection using filter technique

The process is similar to that using wavelet transform. The differences are:

(1) Instead of wavelet decomposition for removing the low frequency normal operation signal, a second order Butterworth high pass filter was used. Its cut off frequency was optimized at $f \mathrm{c}=$ $10 \mathrm{kHz}$ to keep as much as possible fault information in high frequency range.

(2) The output of the high pass filter is a single one dimensional time vector, not as in the wavelet decomposition, where multiple vectors are obtained.

The fault features obtained using both methods for different fault occurrence rates are compared in Figure 11. It shows significant advantages of the wavelet method over the traditional filter method.

For the fast changing faults, the faults were expressed with both linear change of the feature magnitude and much sharper pulses in the wavelet method. In Figure 11a the pulse peak in the wavelet method reached about $80 \mathrm{~mA}$ from the base line, while the filter method only showed a peak value of about 10 $\mathrm{mA}$.

For the slow changing faults, shown in Figure 11a and $b$, although no significant peaks were observed in the higher decomposition levels using wavelet transform, the features in the first level were still clearer than that obtained with the filter method. In the wavelet method, the features showed a difference from 55 to $72.5 \mathrm{~mA}$ with a relative change of about $32 \%$, while the filter method gave a difference from 34 to $42.5 \mathrm{~mA}$ with a difference of only about $25 \%$.
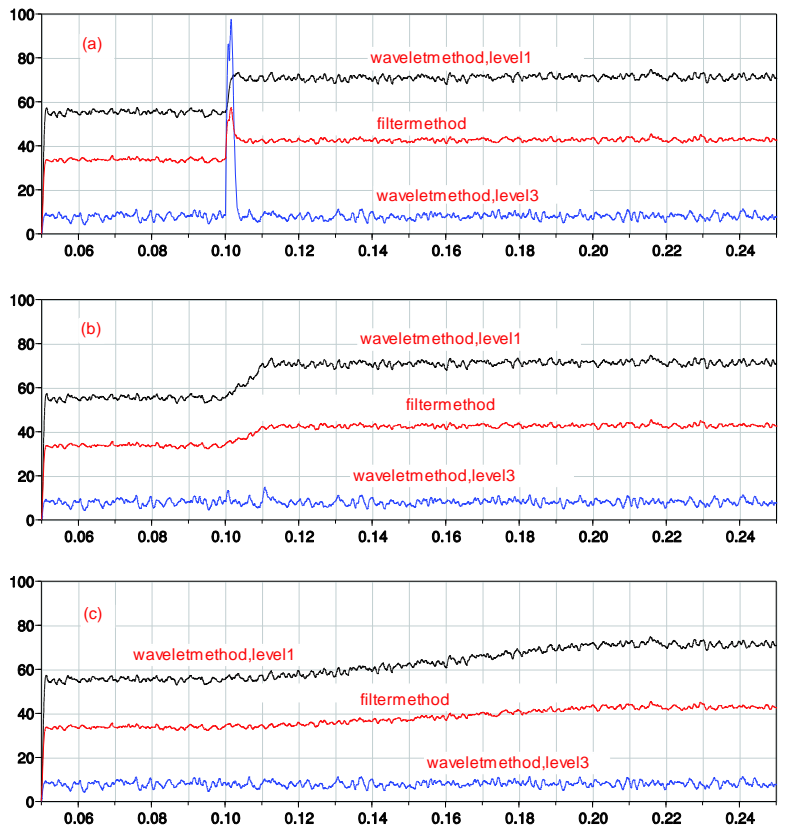

Figure 11: Comparison of the fault features between wavelet method and filter method for (a) $1 \mathrm{~ms}$, (b) $10 \mathrm{~ms}$ and (c) $100 \mathrm{~ms}$ fault occurrence rate (black solid line --- level 1 wavelet; blue dashed line --- level 3 wavelet; red solid line --- filter method)

\section{Conclusion and discussion}

This work described a method for early fault detection of important electric components in power supply systems for more electric aircraft (MEA) using wavelet transform. The special properties of wavelet transform suit this method well for changing signal analysis. The simulation study using Modelica under the environment Dymola illustrated its superior feasibility for the detection of fast changing faults, which was significantly better than the traditional filter technique. For the slow changing faults, the wavelet method also gave a significant feature and provided clearer information than the filter technique. Based on these advantages, the wavelet fault detection method is expected to achieve satisfied detection of early faults.

In this study, a specific wavelet library for Modelica is being developed, which possesses the basic functionality of wavelet analysis, including wavelet transform and inverse transform, wavelet decomposition and reconstruction for multiresolution analysis, and other related functions. The work proved the feasibility of the implementation of wavelet analysis in Modelica.

More work is being done in this topic, including further development of the Modelica wavelet library and experimental study of the fault detection with a 
real buck inverter. For a real system, detailed optimization of the fault detection strategy has to be carried out, such as trying other wavelet functions, observing more changing rates, studying the detection with expanded fault ranges, and considering the faults in more electrical parts.

\section{Acknowledgement}

The authors thank the support from the Clean-Sky Joint Undertaking through the Grant Agreement No. 296369 (Project MoMoLib) within the Seventh Framework Programme of the European Union.

\section{References}

[1] C. Schallert, A. Pfeiffer and J. Bals. Generator power optimisation for a more-electric aircraft by use of a virtual iron bird, $25^{\text {th }}$ Internaltional Congress of the Aeronautical Sciences, 2006.

[2] M.R. Kuhn, Y. Ji, D. Schröder, Stability studies of critical DC power system comonent for More Electric Aircraft using $\mu$ sensitivity. Proc. of the $15^{\text {th }}$ Mediterranean Conference on Contro \& Automation, 2007.

[3] J. Gertler. Fault Detection and Diagnosis in Engineering Systems. Marcel Dekker, 1998.

[4] J. Chen and P.Patton. Robust Model-based Fault Diagnosis for Dynamic Systems. Kluwer Academic Publishers, 1999.

[5] S. X. Ding. Model-based Fault Diagnosis Techniques: Design Schemes, Algorithms and Tools. Springer Berlin, 2008.

[6] K. Swarup and H. Chandrasekharaiah. Fault detection and diagnosis of power systems using artificial neural networks. 1st international forum on application of neural networks to power system, 1991.

[7] M. Aldeen and F. Crusca. Observer-based fault detection and identification scheme for power systems: Generation, Transmission and Distribution. IEE Proceedings, vol. 153, pp.71-79, 2006.

[8] Y. Ji and J. Bals. Multi-Model Based Fault Detection for the Power System of More Electric Aircraft. Proceedings of the 7th Asian Control Conference, Hong Kong, China, Aug. 27-29, 2009.
[9] Y. Ji, J. Bals, Application of model detection techniques to health monitoring for the electrical network of More Electric Aircraft, International Conference on Electrical Engineering and applications, 2009.

[10] S. Mallat (2009): A wavelet tour of signal processing - the sparse way. Amsterdam: Elsevier.

[11] H. T. Zhang, Q. An, et al. Fault Detection Wavelet Fractal Method of Circuit of ThreePhase Bridge Rectifier. International Conference on Intelligent System Design and Engineering Application (ISDEA), 2010, S. 725729.

[12] V. Prasannamoorthy, N. Devarajan, et al. Wavelet and Fuzzy Classifier Based Fault Detection Methodology for Power Electronic Circuits. International Conference on Process Automation, Control and Computing (PACC), 2011, S. 1-6.

[13] T. Buente, A. Sahin, and N. Bajcinca, Naim. Inversion of Vehicle Steering Dynamics with Modelica/Dymola. Proceedings of the 4th International Modelica Conference 2005, S. 319-328.

[14] J. Bals, Y. Ji, M. R. Kuhn, C. Schallert, Model based design and integration of More Electric Aircraft systems using Modelica. Moet forum at European power electronics conference and exhibition, 2009.

[15] Y. Ji, J. Bals, A Modelica signal analysis tool towards design of More Electric Aircraft, ICIAE, 2010.

[16] E. Jacobsen and R. Lyons. The sliding DFT. Signal Processing Magazine, vol. 20, issue 2, pp. 74-80, March 2003.

[17] I. Daubechies. Ten Lectures on Wavelets. SIAM 1992. 
